\title{
EXPERIMENTAL STUDY OF THERMO-ACOUSTIC INSTABILITY TRIGGERING IN A STAGED LIQUID FUEL COMBUSTOR USING HIGH-SPEED OH-PLIF
}

\author{
Antoine Renaud* \\ Shigeru Tachibana \\ Aeronautical Technology Directorate \\ Aeronautical Technology Directorate \\ Japan Aerospace Exploration Agency Japan Aerospace Exploration Agency \\ (JAXA) \\ (JAXA) \\ Tokyo, Japan \\ $\&$ \\ Email: tachibana.shigeru@jaxa.jp \\ Faculty of Science and Technology \\ Keio University \\ Tokyo, Japan
}

\section{Shuta Arase \\ Takeshi Yokomori}

Faculty of Science and Technology

Keio University

Tokyo, Japan

Email: yokomori@mech.keio.ac.jp

Email: antoine.renaud@centralesupelec.fr

\begin{abstract}
A staged injector developed by JAXA and fueled with kerosene is studied in a high-pressure combustion experiment. With a stable pilot fuel flow rate, the fuel flow rate in the main stage is progressively increased. A high-speed OH-PLIF system is used to record the flame motion at 10,000 fps. In the beginning of the recording, the flame behavior is dominated by relatively low-frequency rotation due to the swirling motion of the flow. These rotational motions then coexist with a thermo-acoustic instability around $475 \mathrm{~Hz}$ which increases the amplitude of the pressure fluctuations inside the chamber. DMD analyses indicate that this instability is associated with a widening of the flame occurring when the pressure fluctuations are the highest, giving the instability a positive feedback. The instability frequency then abruptly switches to $500 \mathrm{~Hz}$ while the mode shape remains
\end{abstract}

${ }^{*}$ Address all correspondence to this author. 
the same. This frequency change is studied using time-frequency analysis to highlight a change in the feedback mechanism characterized by a modification of the time delay between pressure and heat release fluctuations.

\title{
NOMENCLATURE
}

\author{
DMD Dynamic Mode Decomposition \\ PLIF Planar Laser-Induced Fluorescence \\ RMS Root Mean Square \\ SVD Singular Value Decomposition
}

\section{INTRODUCTION}

Increasingly stringent pollutant emission regulations lead aircraft engine manufacturers to move away from traditional diffusion flame based combustion systems [1]. To decrease nitrogen oxides (NOx) emissions, lean premixed combustion presents interesting properties. Such flames tend however to be prone to thermo-acoustic instabilities that can cause catastrophic damage to the engine through very high amplitude pressure oscillations. This issue has been an important research topic over several decades [2,3] but remains to be fully solved because of its inherent complexity. Indeed, by involving on a pressure-driven feedback on the flame, thermo-acoustic instabilities link together several physical processes like fuel-air mixing (including liquid fuel atomization and vaporization when needed) or vortex-flame and acoustic-flame interactions. In real engines, these processes happen in elevated pressure and temperature conditions with generally complex geometries.

In order to increase flame stabilization, staged injectors have been developed following the idea that injecting a small amount of fuel upstream of the main flame can create a hot region filled with burnt gases to have an anchoring effect and prevent high-amplitude oscillations [4]. The price to pay is however an increase in complexity with additional swirlers, fuel feeding lines and fuel injection and atomization techniques. Because of the numerous interplay that can arise, it is best to study these injectors in configurations as close to the industrial applications as possible. This however requires the use of powerful tools whether from a numerical or experimental point of view.

In fluid mechanics, and in the combustion field in particular, laser diagnostics have been extensively used to obtain flow field parameters with minimal intrusiveness [5]. As experimental conditions become harsher (high pressure and temperature, thermo-acoustic instabilities), setting laser diagnostics however start to present technical difficulties explaining why their use in somewhat realistic conditions is limited. For the analysis of instabilities in gas turbine type combustors at elevated pressure, mostly low frequency laser diagnostics such as Particle Image Velocimetry (PIV) or Planar Laser-Induced Fluorescence (PLIF) have been used [6-9]. Boxx et al. [10], used $3 \mathrm{kHz}$ simultaneous PIV/OH-PLIF measurements to study a $388 \mathrm{~Hz}$ thermo-acoustic instability in a natural gas fueled combustor at 5 bars. Tachibana et al. [11] highlighted the existence of a strong thermo-acoustic instability in a kerosene fueled aeroengine 
combustor using low repetition rate OH-PLIF among other techniques. This work, using the same experimental setup, takes advantage of $10 \mathrm{kHz}$ OH-PLIF recordings to study the transient behaviour leading to the appearance of the instability.

As the fuel flow rate in the main stage of the injector is increased, the combustor, originally in a quiet steady state, starts to exhibit thermo-acoustic instabilities of increasing amplitude. After presenting the injector, the diagnostic techniques and the advanced postprocessing tools required for the analysis of such phenomena, focus is put on three moments of the experiment presenting different levels of pressure fluctuations. The existence of axial and rotational fluctuations is studied and explanations for their origin are proposed.

\section{EXPERIMENTAL CONDITIONS}

\section{Staged combustor}

The measurements were carried out at the JAXA high temperature and high pressure combustion test facility. The test rig can operate at up to $1 \mathrm{MPa}$ and elevated temperature (between 600 and $1273 \mathrm{~K}$ ). The single-sector combustor shown in Fig. 1 is installed in the pressure casing of the test rig. The length of the combustion chamber is $190 \mathrm{~mm}$ with an upstream part equipped with quartz windows for optical access. The downstream part is a two dimensional converging nozzle with multiple small holes for film cooling.

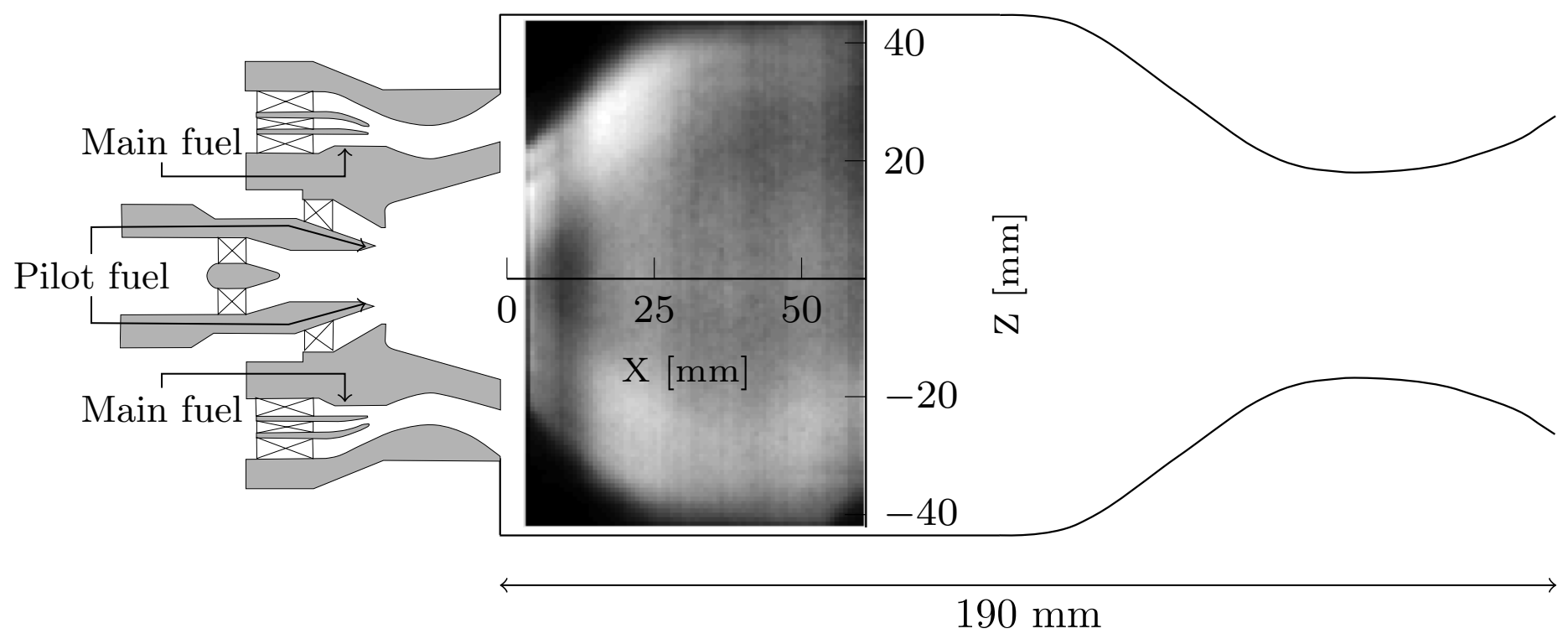

FIGURE 1. SCHEMATICS OF A RADIAL CUT OF THE STAGED INJECTOR AND THE SINGLE SECTOR COMBUSTION CHAMBER. A MEAN OH-PLIF IMAGE IS ADDED TO HIGHLIGHT THE REGION OF INTEREST.

A low-NOx staged injector is mounted on the combustion chamber. Designed by Yamamoto et al. $[12,13]$, it is composed of two stages with multiple concentric axial swirlers. The pilot stage, equipped with two contra-rotating swirlers and an air-blast fuel atomizer, aims at creating a stabilizing region inside its cup to provide anchoring for the main flame. The goal of the main stage is on the contrary 
to provide a good lean mixture of air and fuel to effectively reduce NOx emissions. For this purpose, three contra-rotating swirlers are used to provide a high-shear environment inside which fuel is injected through ten holes with a $0.5 \mathrm{~mm}$ diameter and impinges on a annular pre-filmer. Further information on the experimental setup and on the injector can be found in previous publication [11-13].

In the present work, the setup is operated at a fixed temperature of $760 \mathrm{~K}$ and a pressure of $0.7 \mathrm{MPa}$. The total air flow rate is $364 \mathrm{~g} / \mathrm{s}$ with $225 \mathrm{~g} / \mathrm{s}$ actually going through the injector and the remaining $139 \mathrm{~g} / \mathrm{s}$ being used for the combustion chamber cooling. In the pilot stage, a constant $1.8 \mathrm{~g} / \mathrm{s}$ flow rate of kerosene is injected while the main stage kerosene injection is varied from 5.5 to $6.5 \mathrm{~g} / \mathrm{s}$, triggering thermo-acoustic oscillations from an originally stable state.

\section{Measurement parameters}

The main diagnostic in this study is time-resolved OH-PLIF. A high-speed Nd-YAG laser (Edgewave IS-400-2-L) pumps a Rhodamine dye laser (Sirah Credo-DYE-Amp-SH-FE) at $10 \mathrm{kHz}$ to obtain a beam at $283.6 \mathrm{~nm}$. After a set of sheet forming optics, the light is sent vertically into the combustion chamber in order to illuminate the X-Z plane, as shown in Fig. 1.

The fluorescence emission of $\mathrm{OH}$ is recorded with a high-speed camera (Phantom V2011) equipped with an image intensifier (Lambert HiCATT 25D), a $45 \mathrm{~mm}$, f:1.8 UV lens (Sodern) and a 310 $\pm 10 \mathrm{~nm}$ bandpass filter (Asahi MZ0310) to limit the influence of other signals like the fluorescence of the aromatics in the fuel. The frame rate is $10,000 \mathrm{fps}$ and the image intensifier gate is set at $60 \mathrm{~ns}$ to avoid a detrimental effect of $\mathrm{OH}^{*}$ spontaneous emission. The final $70 \times 90 \mathrm{~mm}$ region of interest is imaged with a pixel pitch of $0.18 \mathrm{~mm} /$ pixel, however for noise and processing time reduction reasons, $4 \times 4$ pixels bins are used for the analysis. More than 47,000 images were recorded in total and an example of an instantaneous image after correction of the laser sheet profile is shown in Fig. 2. Even though the image is a bit noisy and prevents a fine analysis of flame wrinkling, it still enables to separate the fresh and burnt gas regions. The flame region show high signal intensity while the center of the image is made of recirculating burnt gases with slightly lower intensity values. It is possible to detect the regions of heat release by computing the gradient intensity of the OH-PLIF images since steep variations of intensity correspond to a passage from unburnt to burnt gases (meaning a flame front) while more gradual variations correspond to equilibrium and mixing in the burnt gases [14]. In this study, this image gradient is obtained with the commonly used Sobel operator.

The pressure oscillations inside the combustion chamber are measured thanks to a pressure transducer (Meggitt Vibro-Meter CP211). Integrated $\mathrm{OH}^{*}$ chemiluminescence is also recorded with a photomultiplier. The signals from these sensors, as well as the laser, camera and intensifier gate trigger signals are recorded using a multi-channel recorder (TEAC WX-7000) at a frequency of $200 \mathrm{kHz}$. Electric noise at $50 \mathrm{~Hz}$ in the pressure transducer signal is removed using a $2 \mathrm{~Hz}$-wide notch filter with a $10 \mathrm{~dB}$ attenuation before processing. 


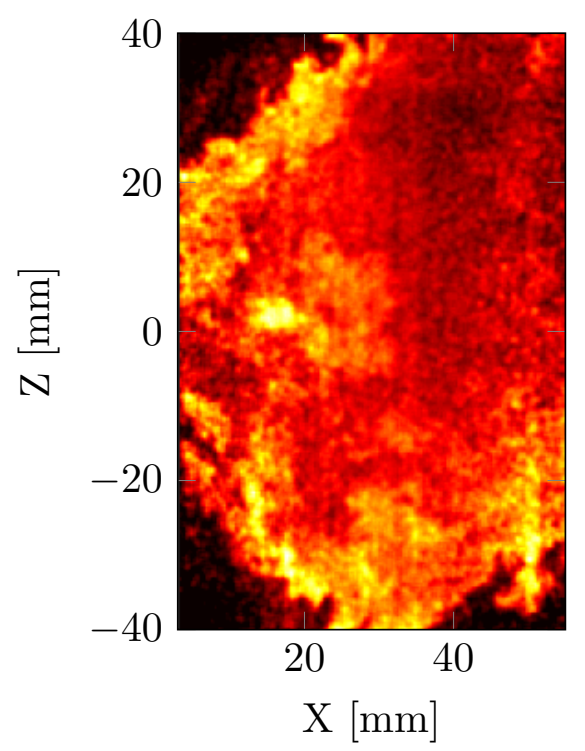

FIGURE 2. INSTANTANEOUS IMAGE FROM THE OH-PLIF RECORDINGS.

\section{Dynamic Mode Decomposition}

Time-resolved data obtained from high-speed OH-PLIF contain a lot of information but also a lot of unwanted content. Particular post-processing methods are thus needed to extract useful material, namely spatial structures or frequencies in the flame response. Dynamic Mode Decomposition (DMD) is a particularly well-suited tool for this purpose and thus extensively used in this study. DMD is a linear analysis technique which extracts mono-frequential modes from a set of time-resolved data [15,16]. This characteristic makes it very useful to analyze thermo-acoustic oscillations since they generally offer a narrow frequency response.

Under the assumption of a linear link between the data at one instant and the next one, the main idea is to study the properties of the operator $A$ that makes this link. For a recording of $\mathrm{N}$ snapshot, placed in a column matrix, the relationship between a sub-matrix of the first to the penultimate snapshot, $V_{1}^{N-1}$, and the sub-matrix of the second to the last snapshot, $V_{2}^{N}$, can be written with the operator $A$ as:

$$
V_{2}^{N}=A V_{1}^{N-1}
$$

Directly computing $A$ from the original set of data is however impossible therefore several algorithms can be used to obtain an approximation of the eigenvalues and eigenvectors of $A$ [17]. In the present work, a SVD of $V_{1}^{N-1}$ is performed to compute a matrix $F$ which is 
the projection of $A$ on the POD modes of $V_{1}^{N-1}$. Using $U \Sigma W^{*}$ as the SVD of $V_{1}^{N-1}, F$ is computed using the following expression:

$$
F=U^{*} V_{2}^{N} W \Sigma^{-1}
$$

The DMD modes and their frequencies are then obtained from the eigendecomposition of $F$. They depend on both spatial and temporal coordinates and can be written as follows:

$$
M(x, t)=K(x) e^{i \phi(x)} e^{(a+i \omega) t}
$$

where $K(x)$ is a spatially dependent oscillation amplitude, $\phi(x)$ the original phase of the oscillation and $e^{(a+i \omega) t}$ the oscillation itself. This way of writing makes it easy to separate the spatial content from the temporal content of the mode. To determine which modes are important in the data, a DMD spectrum can be used by plotting each mode's norm versus its frequency. In the present work, the mode norm is the $\mathrm{L}^{2}$-norm of the mode taken at half the duration of the recording.

The information contained in the spatial part of the mode corresponds to two quantities: the amplitude $K(x)$ and the phase $\phi(x)$. Both are important as $K(x)$ allows to identify the regions that are strongly oscillating while $\phi(x)$ provides the relationship between these regions, enabling to identify convective motions for example. It is therefore interesting to trace these two quantities in the same image and, for this purpose, a particular colormap has to be used. This colormap, shown in Fig.3, allows to represent the amplitude of the mode $K(x)$ as light intensity and the phase $\phi(x)$ in color. It is extensively used in this study to represent the DMD modes. Because the phase $\phi(x)$ is relative, all modes are traced with an arbitrary phase reference of 0 at $\mathrm{X}=10 \mathrm{~mm}$ and $\mathrm{Z}=20 \mathrm{~mm}$.

\section{BURNER BEHAVIOR}

The experiment consists in increasing the main fuel flow rate from $5.5 \mathrm{~g} / \mathrm{s}$ to $6.5 \mathrm{~g} / \mathrm{s}$ while keeping the pilot constant. Even though no high-speed recording of the fuel flow rate is available, existing logs show that most of the increase occurs during the first second of the recording. During this increase, the pressure fluctuations inside the chamber grow to reach a stable limit cycle after about 1 second, as shown by the pressure transducer signal in Fig. 4. The analysis focuses on three parts of the recording. From 0 to 0.5 second (part 1 in Fig. 4), no thermo-acoustic instability can be detected and the pressure fluctuations correspond to $3.7 \mathrm{kPa}$ RMS. From 0.5 to 1 second (part 2 in Fig. 4), the pressure fluctuations reach $5.5 \mathrm{kPa}$ RMS with a clear peak in the spectrum. Finally, from 1 to 1.5 second (part 3

in Fig. 4), a thermo-acoustic instability is clearly developed and the pressure fluctuations are measured at $9.5 \mathrm{kPa}$ RMS. They then keep this level until the end of the recording 3 seconds later. 


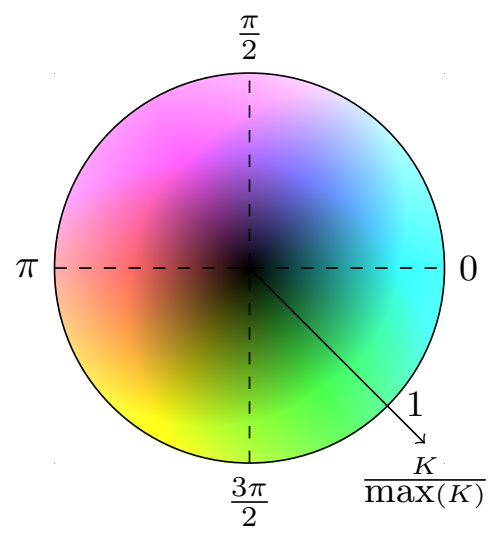

FIGURE 3. COLORMAP USED FOR THE REPRESENTATION OF THE DMD MODES.

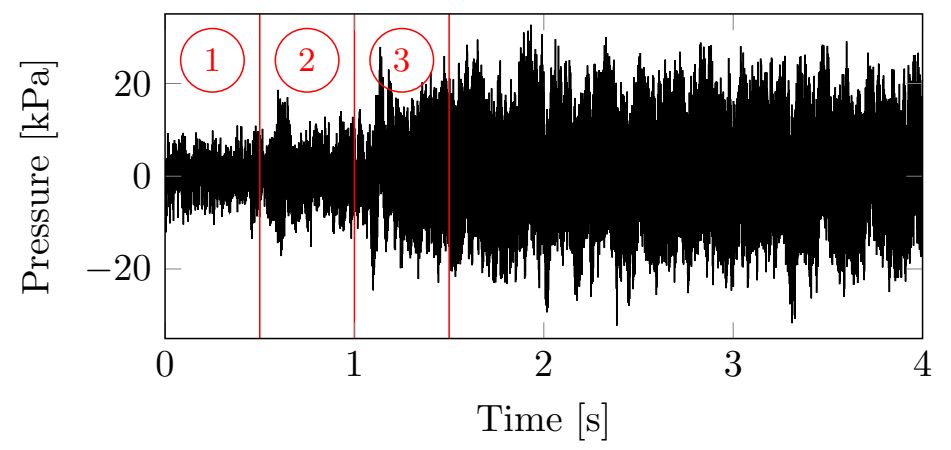

FIGURE 4. SIGNAL FROM THE PRESSURE TRANSDUCER HIGHLIGHTING THE 3 STUDIED PARTS.

\section{Before onset of instability}

Before the onset of the instability, the pressure fluctuations signal trace shows no particular frequency response and moderate levels. This however does not necessarily means that the flame is completely stable and to study the flame dynamics, the OH-PLIF recording is used. Figure 5 shows the results of the DMD post-processing of the gradient of the OH-LIF signal between 0 and $0.5 \mathrm{~s}$. This represents 5000 snapshots, decomposed into 5000 modes. The norm of the modes with frequencies below $2000 \mathrm{~Hz}$ is shown on the spectrum on the left of Fig 5. Apart from the low frequency modes corresponding to the average signal and slow fluctuations, a peak at $183 \mathrm{~Hz}$ can be seen dominating the noise.

The shape of the mode corresponding to this frequency is drawn on the right of Fig. 5 using the colormap of Fig. 3. The signal is relatively symmetric in intensity but the phase values indicate that the top and bottom parts of the flame are in phase opposition. This means that while the combustion is more intense at the top, the bottom is less active and conversely.

Based on the shape of the mode, two explanations for its origin can be proposed. The first one is the effect of the excitation of a transversal mode of the chamber, sometimes referred to as "screeching" [18]. This is however highly unlikely since, given the dimensions 
$183 \mathrm{~Hz}$
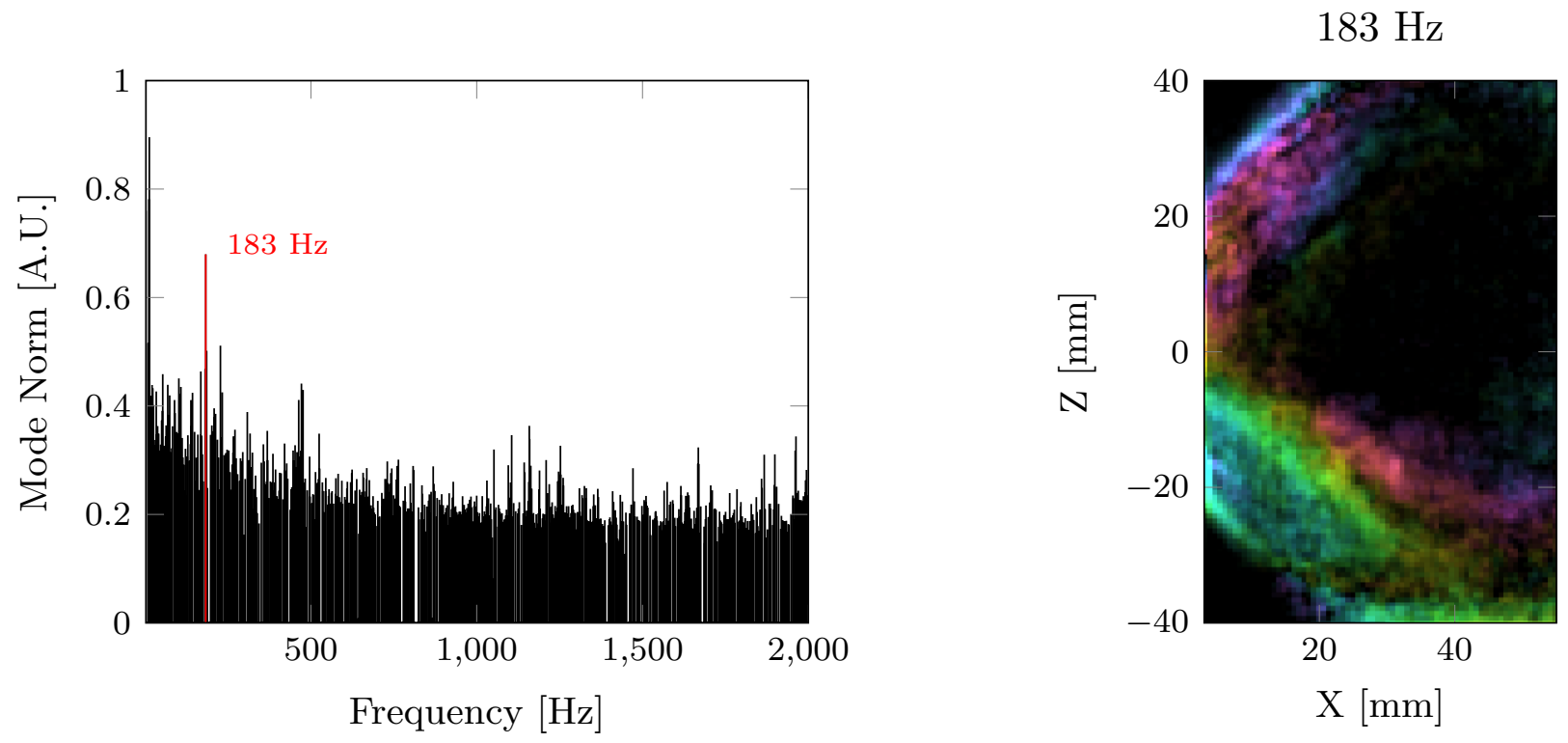

FIGURE 5. RESULTS OF THE DYNAMIC MODE DECOMPOSITION OF THE OH-PLIF GRADIENT BETWEEN T=0 S AND T=0.5 S.

of the chamber, such a mode would have a frequency well above $1 \mathrm{kHz}$. A second explanation for the mode shape seen in Fig. 5 is that it corresponds to the radial cut of a rotating structure due to the swirling motion of the flow. Rough calculations based on the bulk velocity and angles of each swirler tend to show that the rotational frequencies associated with the pilot swirlers are above $500 \mathrm{~Hz}$ while the main swirler ones are around $250 \mathrm{~Hz}$. From this approach, it thus looks like this mode is to be linked with the main swirl more than with the pilot swirl. This is to be expected since most of the air flow goes through the main stage to enter the chamber. This rotational and convective origin of the mode can also explain why it has no impact on the measured global pressure fluctuations.

\section{Appearance of instability}

Focus is now put on the data gathered between 0.5 and 1 second. During this part, the pressure fluctuations reach $5.5 \mathrm{kPa}$ RMS, about 1.5 times their value in the first part. The PSD of the pressure signal is estimated using Welch's method with a Hamming window and $50 \%$ overlap for a final frequency resolution of $5 \mathrm{~Hz}$. The resulting spectrum is plotted in Fig. 6.

Apart from a clear peak around $475 \mathrm{~Hz}$, the spectrum in Fig. 6 shows no visible spectral structure. This indicates that most of the recorded pressure fluctuations are created at this frequency. It corresponds to the signature of a thermo-acoustic instability developing inside the combustor

To study this instability from the flame dynamics point of view, the OH-PLIF recordings are used. The 5000 images taken between 0.5 and $1 \mathrm{~s}$ are post-processed with DMD as was done for the recording between 0 and $0.5 \mathrm{~s}$. The results are shown in Fig. 7 . The DMD spectrum between 0 and $2000 \mathrm{~Hz}$ is plotted at the center of the figure. Two peaks can be identified, being stronger than the baseline.

The first peak is at $184 \mathrm{~Hz}$ which corresponds to the same frequency as the only observed peak in Fig. 5. It is thus reasonable to 


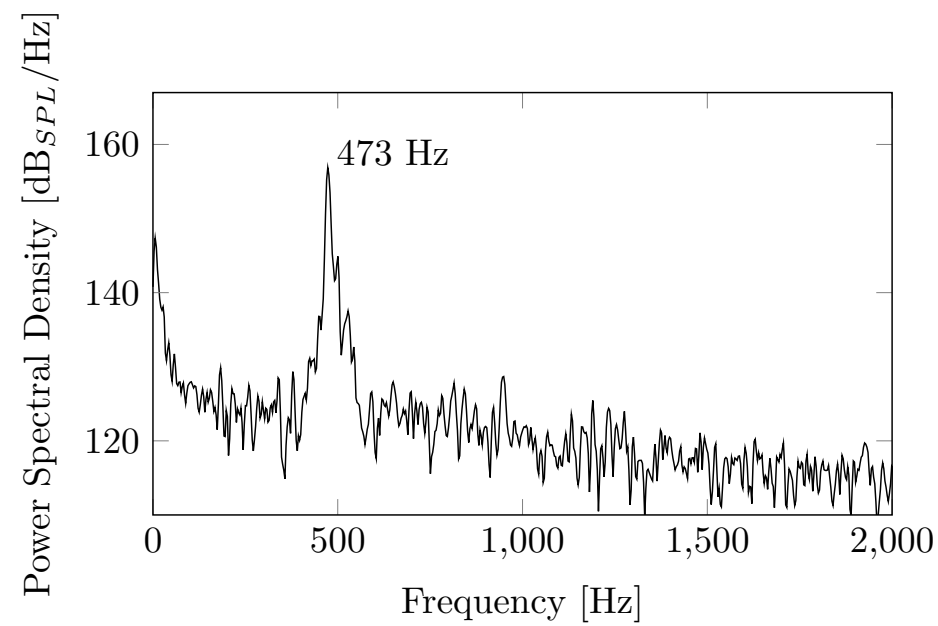

FIGURE 6. POWER SPECTRAL DENSITY OF THE PRESSURE TRANSDUCER SIGNAL BETWEEN T=0.5 S AND T=1 S.

$184 \mathrm{~Hz}$

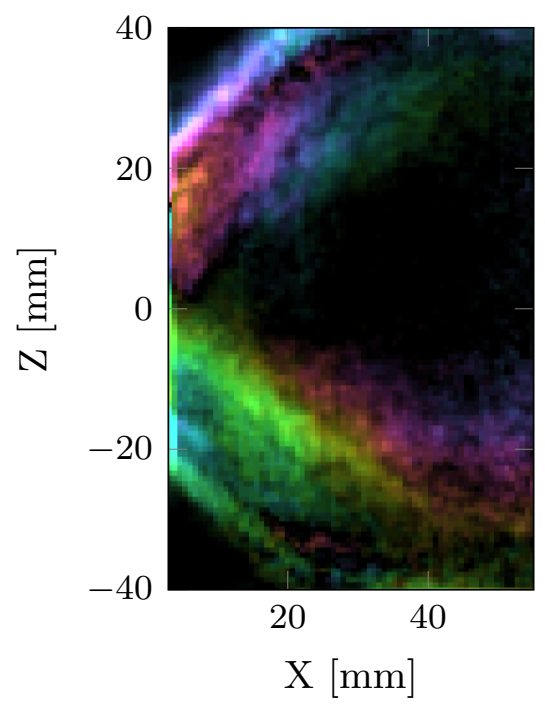

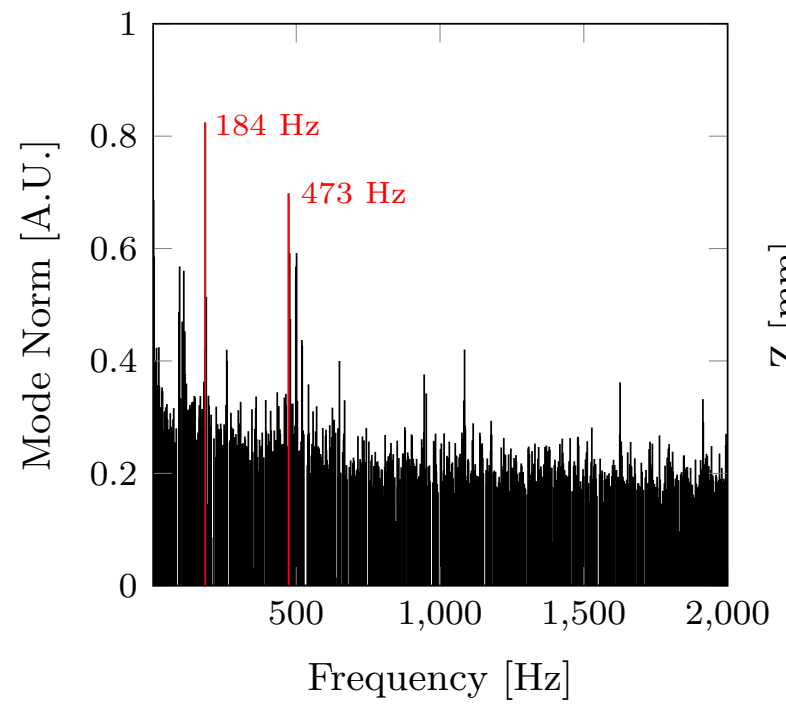

$473 \mathrm{~Hz}$

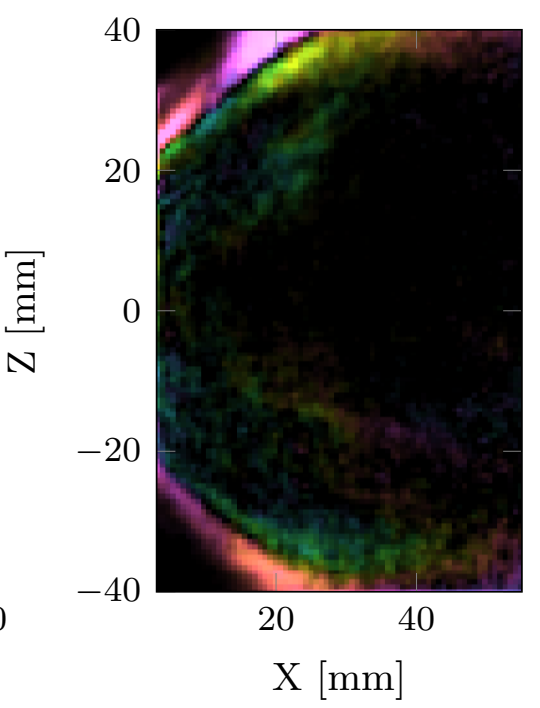

FIGURE 7. RESULTS OF THE DYNAMIC MODE DECOMPOSITION OF THE OH-PLIF GRADIENT BETWEEN T=0.5 S AND T=1 S.

consider that they correspond to the same phenomenon and the associated modes are expected to be completely similar. The shape of the mode at this frequency for the recording between 0.5 and $1 \mathrm{~s}$ is shown on the left of Fig. 7. As expected, it corresponds both in phase and amplitude to the mode observed between 0 and $0.5 \mathrm{~s}$. It is thus also the trace of a disturbance of the flame due to rotational motions.

The second peak of the DMD spectrum at the center of Fig. 7 is at $473 \mathrm{~Hz}$. This is the same frequency as the peak detected in the pressure transducer signal, indicating that it corresponds to the thermo-acoustic instability. The mode corresponding to this frequency is shown on the right of Fig. 7 and isolates the flame fluctuations due to the thermo-acoustic instability. At first sight, it is clear that it is 
completely different from the one at $184 \mathrm{~Hz}$. The high fluctuation intensity concerns only a limited region on the external parts of the flame. All this region is also in phase with values between $\pi / 2$ and $\pi$ whereas the inner part of the flame with the lower intensity is in complete phase opposition (between $3 \pi / 2$ and $2 \pi$ ). It thus appears from this mode that the flame cone is contracting and expanding at the instability frequency with a higher intensity when the flame is fully opened. This indicates a motion of the flame between the pilot and the main region across the small recirculation zone created by the tip of the pilot cup and highlighted in previous LES computations [11]. As the flame opens up, its surface increases and, assuming flame wrinkling stays constant, the heat release also increases. In order to sustain the thermo-acoustic instability, this excess of heat release has to be in phase with pressure. This type of flame motion can be obtained when the instability creates swirl number fluctuations [19].

To analyze the relationship between pressure fluctuations and heat release fluctuations, the Rayleigh criterion is commonly used. It corresponds to the integral of the product of pressure and heat release fluctuations over one cycle of the instability:

$$
R=\frac{1}{\tau} \int_{\tau} p^{\prime}(t) q^{\prime}(t) \mathrm{d} t
$$

where $\tau$ is the oscillation period, $p^{\prime}$ the pressure fluctuations and $q^{\prime}$ the heat release fluctuations. If $R$ is positive, the pressure fluctuations are amplified by heat release whereas if it is negative, damping occurs. When spatially-resolved data is available, it is possible to compute a local Rayleigh index based on the same formula applied at different points of the region of interest. Maps of the local Rayleigh index enable to identify regions where the flame has a positive or negative contribution to the growth of pressure fluctuations. The computation of the local Rayleigh index maps is often performed using a phase averaging of chemiluminescence data based on a pressure transducer reference signal $[11,20]$. In the present work, chemiluminescence recordings are not available and the gradient of OH-PLIF data is used as a representation of heat release instead. LES computations [11] show that the pressure fluctuation amplitude is roughly constant in the combustion chamber, therefore the signal recorded by the pressure transducer can be used as the pressure reference for the whole region of interest.

Computing a local Rayleigh-index also requires a mean to filter out all fluctuations that do not belong to the thermo-acoustic instability. This is generally performed with phase averaging. Here, since the gardient of OH-PLIF data is time-resolved, it is possible to take advantage of the capability of DMD to perform this operation. First, the pressure signal is downsampled to $10 \mathrm{kHz}$ so that each frame of the gradient of OH-PLIF recording can be associated with a pressure value. This value is then treated as an additional pixel in the DMD treatment of the OH-PLIF gradient recording, effectively adding a line to the $V_{1}^{N-1}$ and $V_{2}^{N}$ matrices [21]. After DMD is carried out, the mode corresponding to the studied frequency is extracted and contains both OH-PLIF gradient and pressure signal fluctuations at this frequency as well as the phase relationships between them, as shown by Eq. 3. Using these values, the Rayleigh index can easily be computed. DMD thus avoids the use of phase averaging which requires user input for the choice of the instability 
frequency. Here the frequency appears "naturally" from the information contained in the OH-PLIF gradient recordings and the pressure transducer signal. It must be noted that adding the pressure signal does not visibly impact the results when compared to the DMD of the OH-PLIF gradient alone.

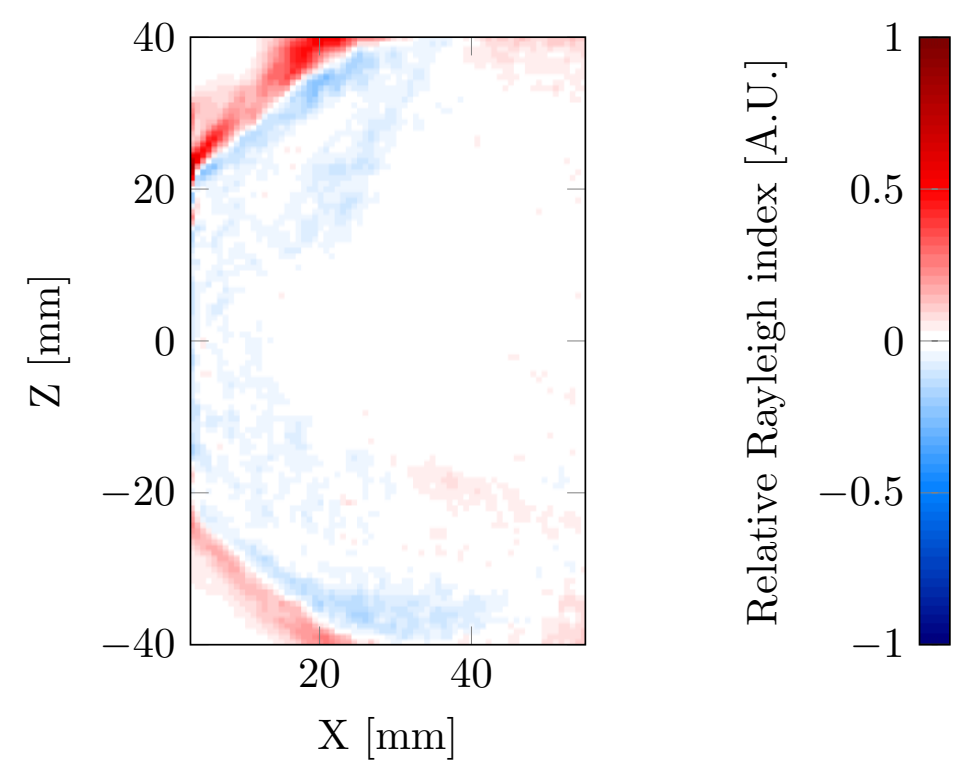

FIGURE 8. RAYLEIGH INDEX BETWEEN T=0.5 S AND T=1 S.

Figure 8 shows a map of the Rayleigh index for the $473 \mathrm{~Hz}$ oscillation. The values are normalized by the maximum that can be obtained in the case of a perfect phase matching which is obtained by multiplying the pressure fluctuation amplitude by the maximum of the fluctuation amplitudes of the gradient of OH-PLIF. In the picture, it can be seen that this maximum value is indeed reached at the top part of the flame around $X=20 \mathrm{~mm}$ and $\mathrm{Z}=40 \mathrm{~mm}$, making of this region a strong contributor to the instability. The Rayleigh index map is very similar to the DMD mode shown in Fig. 7 with high intensities in the external parts of the flame cone and low values inside. This shows that the expansion of the flame is in phase with the pressure cycle. This expansion is also characterized by a larger flame surface area which corresponds to an increase in heat release. The Rayleigh index thus allows to describe the driving mechanism of the thermo-acoustic instability: as the flame widens in phase with the pressure, it adds energy to the cycle and allows the instability to be sustained in spite of the damping present in the setup.

\section{Fully developed instability}

In this section, the third part of the experiment, between 1 and 1.5 second, is analyzed. During this part, a strong thermo-acoustic instability can be identified and the pressure fluctuations reach $9.5 \mathrm{kPa}$ RMS (1.7 times higher than between 0.5 and $1 \mathrm{~s}$ ). This level is kept during the following parts of the recording, up to the end of it after 4.5 seconds. This part is thus representative of the limit cycle 
reached by the thermo-acoustic instability. The PSD of the pressure transducer signal is computed using the same parameters as in the previous section and the resulting spectrum can be seen in Fig. 9. At first sight, it seems quite similar to the one in Fig. 6 but the main

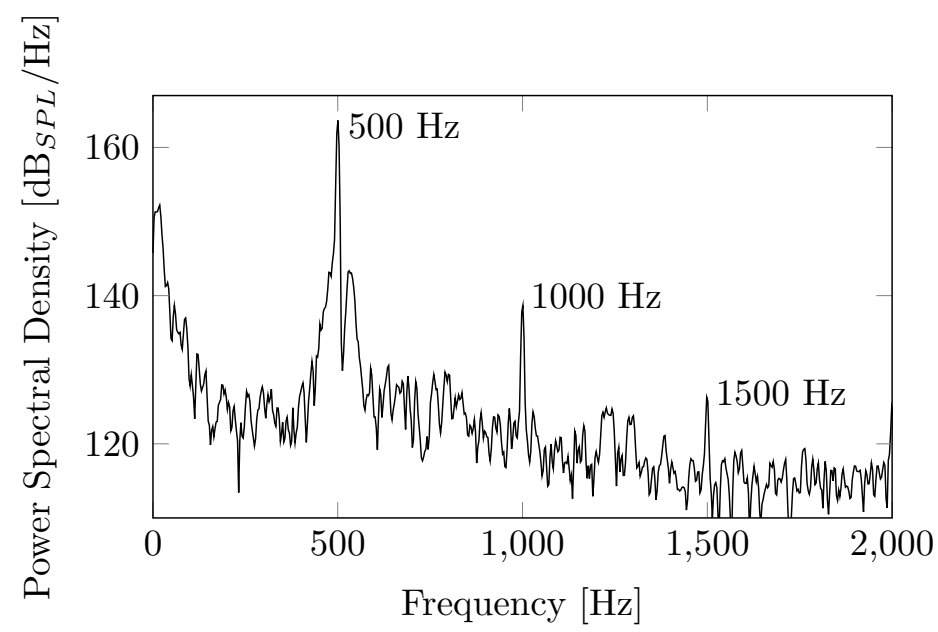

FIGURE 9. POWER SPECTRAL DENSITY OF THE PRESSURE TRANSDUCER SIGNAL BETWEEN T=1 S AND T=1.5 S.

peak is now precisely at $500 \mathrm{~Hz}$ instead of $475 \mathrm{~Hz}$ and the amplitude is now over $160 \mathrm{~dB}$. Furthermore, harmonics can be identified at $1000 \mathrm{~Hz}$ and $1500 \mathrm{~Hz}$. The thermo-acoustic instability is thus not only stronger but also at a different frequency than before. The baseline level seems mostly unaffected and still around $120 \mathrm{~dB}$. A potential explanation for the frequency increase could be an increase of the speed of sound due to a higher temperature in the chamber since this experiment is carried out while the main fuel flow rate (and thus the burner power) increases. A second possibility would be a change in the acoustic mode serving as the basis of the instability. These possibilities are further discussed in the following section of the paper. Because of this frequency change, one could expect a change in the flame shape or dynamics and it is thus necessary to study the flame motions.

Following the method used for the analysis of the previous part of the experiment, DMD is applied to the OH-PLIF gradient data recorded between 1 and 1.5 second. The resulting DMD spectrum is shown on the left of Fig. 10. What is first striking is the disappearance of the peak around $184 \mathrm{~Hz}$ which was attributed to rotational motions of the flame. Previous studies have observed the disappearance of convective rotational structures when the combustor is submitted to high-amplitude pressure oscillations [22-24]. A similar phenomenon might be at stake here. The dominant peak is now at $500 \mathrm{~Hz}$, as expected from the study of the PSD spectrum in Fig. 9. It is really prominent relative to the baseline whose level has not changed compared to Fig. 5 and 7 . A secondary peak corresponding to a $1000 \mathrm{~Hz}$ harmonic can also be observed with roughly half the amplitude of the first peak.

The DMD mode at $500 \mathrm{~Hz}$ is shown on the right of Fig. 10. Despite the change in frequency, this mode is completely similar to the one at $473 \mathrm{~Hz}$ observed in Fig. 7. The external parts of the flame are in phase and the global motion corresponds to an opening 

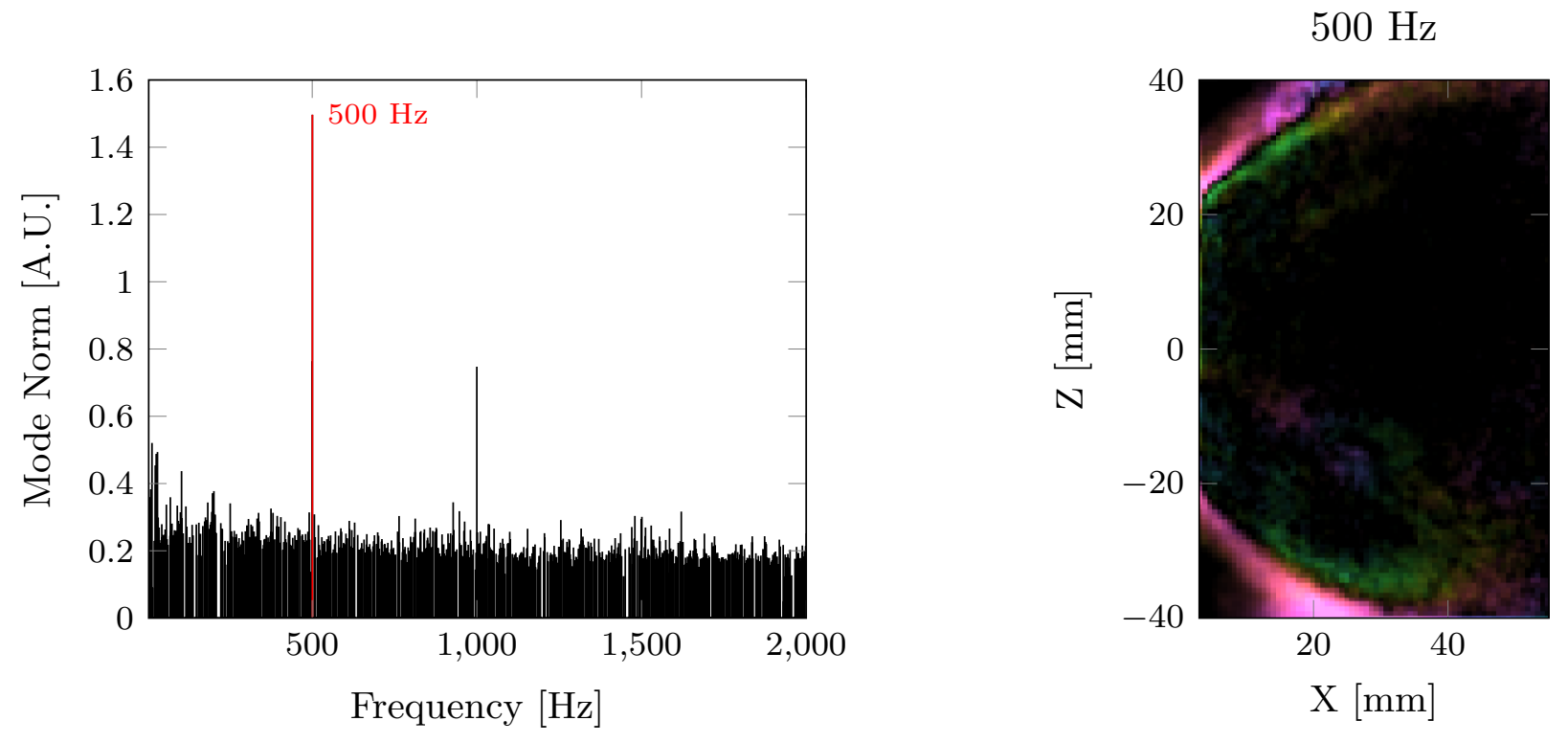

FIGURE 10. RESULTS OF THE DYNAMIC MODE DECOMPOSITION OF THE OH-PLIF GRADIENT BETWEEN T=1 S AND T=1.5 S.

and closing of the flame cone. From the flame motion point of view, there is thus no difference between the $473 \mathrm{~Hz}$ and the $500 \mathrm{~Hz}$ instabilities.

Even though the flame motions are globally the same, a difference may exist in the driving mechanisms of the $473 \mathrm{~Hz}$ and $500 \mathrm{~Hz}$ instabilities. To study this possibility, the Rayleigh index map is traced for the $500 \mathrm{~Hz}$ instability and shown in Fig. 11.

Once again, the Rayleigh index for the $500 \mathrm{~Hz}$ instability is almost identical to the one for the $473 \mathrm{~Hz}$ shown in Fig. 8 . This indicates that for both cases, energy is given to the thermo-acoustic cycle by the widening of the flame during a high-pressure phase. From the flame dynamics point of view, there is thus no discernable difference between the instability at $475 \mathrm{~Hz}$ and the one at $500 \mathrm{~Hz}$. This change of frequency remains however yet to be explained which is the focus of the following section.

\section{CHANGE OF THE INSTABILITY FREQUENCY}

To analyze the change in frequency of the instability, it is necessary to obtain a way to follow the evolution of the frequency with time. This is not an easy task to perform as time and frequency are linked and it is generally impossible to have a perfect precision on both at the same time. Among the numerous methods to obtain time-frequency representations, wavelets and short-term Fourier transform are often used. They enable to obtain a time-frequency map of the signal with specific constraints. In the present study, the thermo-acoustic instabilities are characterized by only one frequency so another method called Hilbert transform is used. Given a 

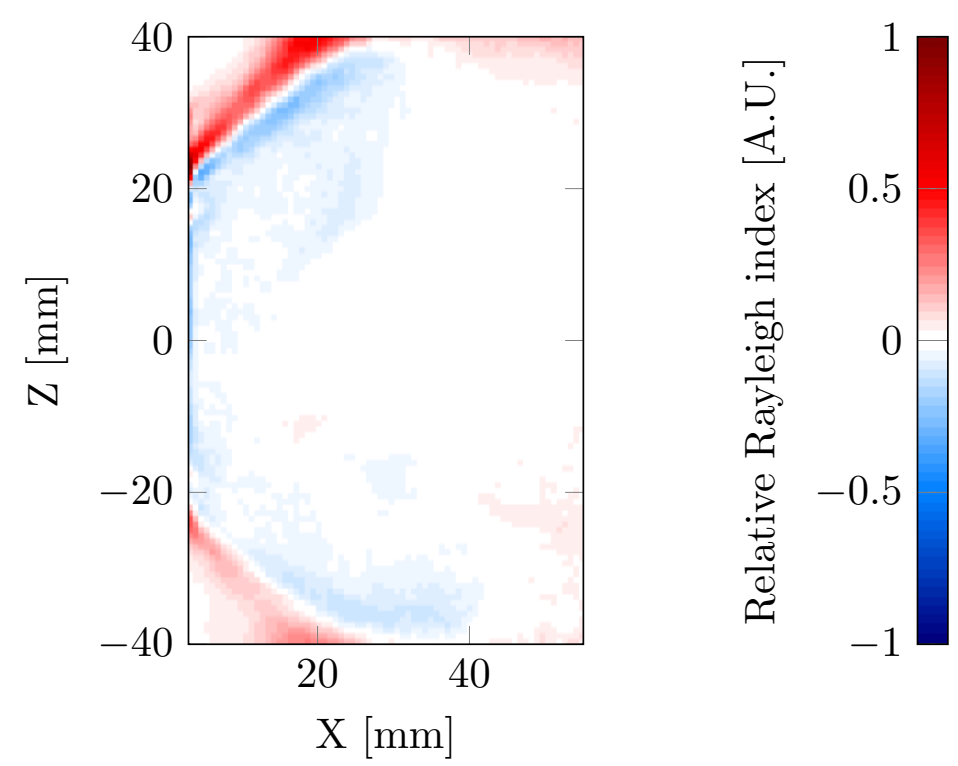

FIGURE 11. RAYLEIGH INDEX BETWEEN T=1 S AND T=1.5 S.

temporal signal $s(t)$, the Hilbert transform is defined as:

$$
H_{s}(t)=\frac{1}{\pi} \int_{-\infty}^{\infty} \frac{s(\tau)}{t-\tau} d \tau
$$

It enables to obtain the analytical signal $s_{a}(t)$ :

$$
s_{a}(t)=s(t)+i H_{s}(t)
$$

which is a complex signal from which it is then possible to extract an instantaneous amplitude $A(t)$ and an instantaneous phase $\phi(t)$ so that $s_{a}(t)=A(t) e^{i \phi(t)}$. If the original signal $s(t)$ is a narrow-band signal, $A(t)$ and $\phi(t)$ can provide useful information on it. In this case, $A(t)$ is the instantaneous amplitude of $s(t)$ and $\frac{d \phi(t)}{d t}(t)$ is its instantaneous frequency. Hilbert transform is thus a very interesting tool to study the thermo-acoustic instability frequency change occurring between $0.5 \mathrm{~s}$ and $1.5 \mathrm{~s}$.

\section{Pressure transducer signal}

As explained before, the information obtained on the instantaneous amplitude and the instantaneous frequency with Hilbert transform is only useful for a narrow-band signal. To apply this processing method to the presure transducer signal, it is thus first necessary 
to remove all unwanted components and noise. Because the band of interest corresponds to the thermo-acoustic instability around 473 or $500 \mathrm{~Hz}$, the raw pressure transducer signal is first filtered with a bandpass filter (center frequency: $487 \mathrm{~Hz}$, bandwidth: $100 \mathrm{~Hz}$ ) using a zero-phase filtering processing to avoid lags. Hilbert transform is then applied to the filtered signal and the resulting instantaneous amplitude and frequency of the pressure signal between $0.5 \mathrm{~s}$ and $1.5 \mathrm{~s}$ are shown in Fig. 12. It must be noted that, to avoid border effects due to the bandpass filtering and the Hilbert transform, a longer window of the pressure signal is actually processed and only its central part between $0.5 \mathrm{~s}$ and $1.5 \mathrm{~s}$ is retained.
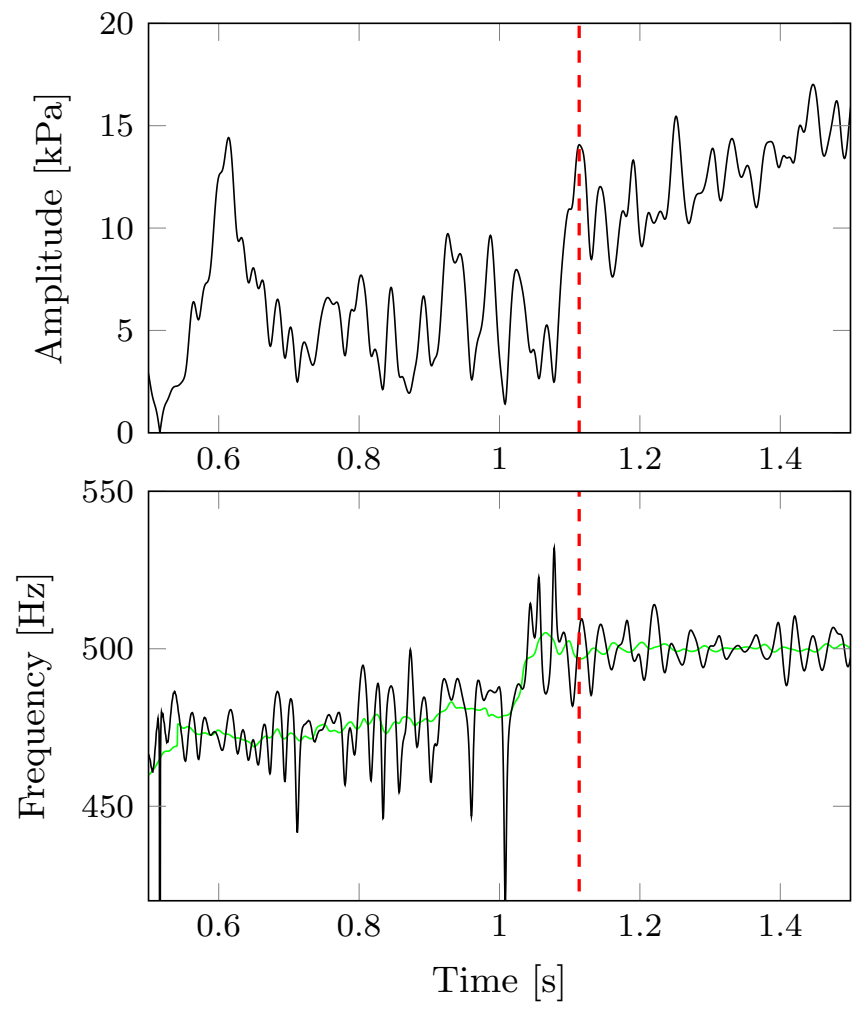

FIGURE 12. INSTANTANEOUS AMPLITUDE AND FREQUENCY FROM THE HILBERT TRANSFORM OF THE PRESSURE TRANSDUCER SIGNAL BETWEEN T $=0.5 \mathrm{~S}$ AND T=1.5 S.

The amplitude plot shows two different regions. First, from $0.5 \mathrm{~s}$ to a bit after $1.1 \mathrm{~s}$, the amplitude is relatively stable around $5 \mathrm{kPa}$. This corresponds to the second part identified in the raw pressure signal and associated with the $473 \mathrm{~Hz}$ instability. Around $1.1 \mathrm{~s}$, a strong increase of more than $5 \mathrm{kPa}$ in the amplitude is visible and the amplitude then steadily rises up to $15 \mathrm{kPa}$. The end of the abrupt rise is shown as a red dashed line and corresponds to $\mathrm{t}=1.11 \mathrm{~s}$.

The previous section showed that the instability frequency is different between $0.5 \mathrm{~s}$ and $1 \mathrm{~s}$ than between $1 \mathrm{~s}$ and $1.5 \mathrm{~s}$. In general, such frequency changes are due to changes in gas temperature which impacts the speed of sound in the system. This mechanism could 
be at stake here since the power of the burner is increased during the recording. To verify this hypothesis, the instantaneous frequency of the pressure signal is shown at the bottom of Fig. 12. A sliding average (10 ms width) of the instantaneous frequency curve is also shown in green on the same graph. At $0.5 \mathrm{~s}$, the frequency is around $465 \mathrm{~Hz}$ and rises steadily to around $480 \mathrm{~Hz}$ at $1 \mathrm{~s}$. This slow frequency increase can be attributed to the previously stated gas temperature increase. At $1 \mathrm{~s}$, however, the frequency abruptly gains about $20 \mathrm{~Hz}$ to stabilize at $500 \mathrm{~Hz}$ and keep this value until $1.5 \mathrm{~s}$ (actually until the end of the recording which is not shown here). This frequency jump occurs at $\mathrm{t}=1.05 \mathrm{~s}$ and is too sudden to be attributed to a temperature change as was the slow increase between $0.5 \mathrm{~s}$ and $1 \mathrm{~s}$. Another interesting feature comes from the comparison between the instantaneous amplitude and instantaneous frequency curves. Indeed, the frequency change occurs before the increase in amplitude and there are more than $50 \mathrm{~ms}$ between the two phenomena. Everything thus looks as though the $500 \mathrm{~Hz}$ instability is a new one that needs time to overcome damping and reach a limit cycle rather than a simple change of the previous $473 \mathrm{~Hz}$ one. In order to obtain more information, it is necessary to look at the instability mechanism by combining the pressure and heat release information.

\section{Pressure and heat release fluctuations}

Thermo-acoustic instabilities stem from the interaction of pressure and heat-release fluctuations. Here, pressure fluctuations are recorded thanks to the pressure transducer and global heat release can be associated to the integrated emission of the $\mathrm{OH}^{*}$ radical. It is thus interesting to follow the evolution of both signals between $0.5 \mathrm{~s}$ and $1.5 \mathrm{~s}$ to analyze the transition from the $473 \mathrm{~Hz}$ to the $500 \mathrm{~Hz}$ instability. This is shown as a phase portrait with the pressure fluctuation on the horizontal axis and the $\mathrm{OH}^{*}$ fluctuations on the vertical axis in Fig. 13. Time evolution is represented as colors changing from blue to yellow.
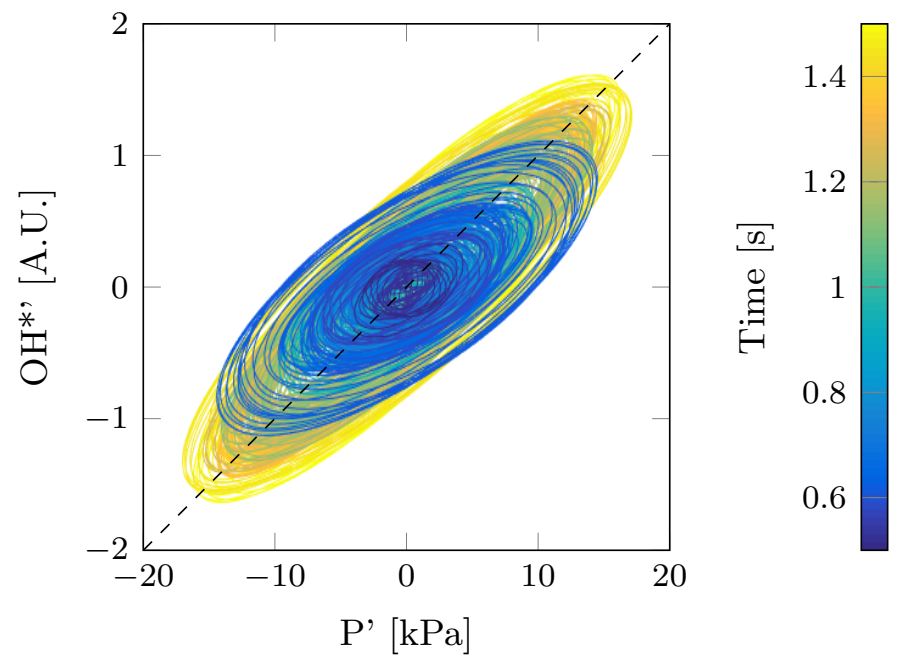

FIGURE 13. PHASE PORTRAIT OF THE PRESSURE AND HEAT RELEASE FLUCTUATIONS BETWEEN T=0.5 S AND T=1.5 S. 
The phase portrait shows ellipses meaning that the pressure and heat release signals are oscillating at the same frequency with a constant phase difference. This phase difference can be estimated by the orientation of the ellipse, the dashed line oriented at $45^{\circ}$ corresponding to a zero phase difference. Two different behaviors can clearly be observed. Before $1 \mathrm{~s}$, the ellipses are more inclined than after. The amplitudes of both pressure and heat release fluctuations are also lower. These differences are obviously associated with the previously observed change in frequency.

To analyze more closely the highlighted change in phase difference, Hilbert transform is used. The pressure fluctuation and $\mathrm{OH}^{*}$ fluctuations signals are bandpass filtered as before and the analytic signal is computed. Instead of looking for an instantaneous frequency however, the instantaneous phase values are used to compute the instantaneous phase difference between heat release (represented by the $\mathrm{OH}^{*}$ signal) and pressure fluctuations. This instantaneous phase difference between 0.5 and $1.5 \mathrm{~s}$ is shown in Fig. 14.

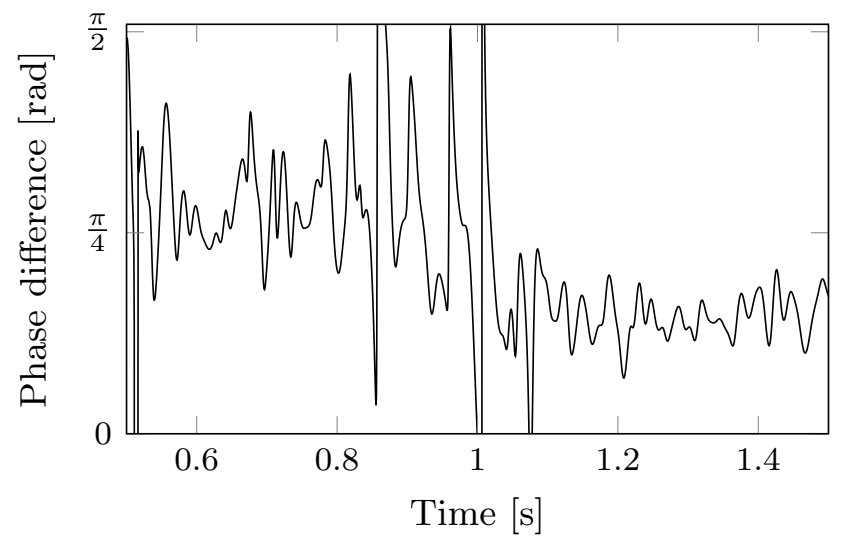

FIGURE 14. PHASE DIFFERENCE BETWEEN THE PRESSURE FLUCTUATIONS AND THE OH* FLUCTUATIONS.

It can first be observed that the phase difference between pressure and heat release is always between 0 and $\frac{\pi}{2}$ which fulfills the Rayleigh criterion and explains why a thermo-acoustic instability at $473 \mathrm{~Hz}$ or $500 \mathrm{~Hz}$ is always present. However, before $1 \mathrm{~s}$, the phase difference is around $\frac{\pi}{4}$ while it decreases to almost $\frac{\pi}{8}$ after. This lower value indicates that pressure and heat release are more in phase which can bring more energy into the thermo-acoustic oscillation. This may be a possible reason for the higher oscillation amplitude after $1 \mathrm{~s}$, as seen in Fig. 12. It can also be seen that the decrease of the phase difference value occurs quite abruptly a bit after $1 \mathrm{~s}$ and is linked to the also abrupt change in frequency observed in Fig 12.

The phase shift between pressure and heat release is linked to the delay necessary for the coupling of the two signals [25]. This delay depends on the coupling mechanism and can for example be associated to convective time scales [26] or vaporization time scales [27] in the case of two-phase combustion. An abrupt change in the time delay as observed in Fig. 14 thus indicates a major change in the coupling mechanism between pressure and heat release. 
Noiray et al. [28] have computed the growth rates of instability modes through non-linear instability analysis. Some modes have a positive growth rate for low amplitude perturbations, reaching zero as the amplitude increases. This corresponds to a linearly unstable with a saturation effect. Other modes start with negative values of the growth rate for low amplitude fluctuations, indicating that they are linearly stable. Their growth rate however reaches positive values for higher amplitudes, showing that they become unstable, before falling back to zero because of saturation. Such modes can thus only be excited if the fluctuation amplitude reaches a threshold. A similar phenomenon might be at stake in the present experiment with the $473 \mathrm{~Hz}$ mode presenting the first type of behaviour and the $500 \mathrm{~Hz}$ mode the second one. The $473 \mathrm{~Hz}$ mode is first excited from low amplitude perturbations and grows to reach a limit-cycle. Then, as the burner power is increased through the main fuel flow rate, more energy can be used for the instability and the threshold of positive growth rate for the $500 \mathrm{~Hz}$ mode is reached. This mode thus grows and, assuming its saturation value is higher than the $473 \mathrm{~Hz}$ one, it completely replaces it, explaining what is seen after $1 \mathrm{~s}$ in Fig. 12. Potential candidates for this non-linear effect could be vortex shedding [29] and liquid fuel fluctuations [30]. The first phenomenon could indeed play a role in the instability even from very low amplitudes at the $473 \mathrm{~Hz}$ frequency. On the contrary, a high pressure fluctuation amplitude is required to start acting on the fuel injection through the main stage. This could be the trigger for the abrupt switch to $500 \mathrm{~Hz}$ and would explain the change in the feedback delay between pressure and heat release. Further information is obviously required to confirm or infirm such a mechanism. For example, measurements of the pressure inside the fuel feeding lines would allow to determine how much the instability has a feedback on the fuel injection. A more theoretical analysis with the present setup appears extremely complex as it requires the possibility of computing the acoustic modes of the burner with its complex geometry and boundary conditions and, most importantly, to obtain a flame describing function [31] of the liquid-fueled, pilot stabilized flame under high-pressure conditions.

\section{CONCLUSION}

Using a realistic aeroengine injector operating under pressurized conditions, triggering of thermo-acoustic instability has been studied with the help of high-speed OH-PLIF recordings. Using the pilot stage with a fixed flow rate to stabilize the flame, the fuel flow rate inside the main stage is progressively increased. First, no particular frequency can be detected in the pressure fluctuations signal. However, when the gradient of the OH-PLIF images (linked to the heat release by the flame) is analyzed using DMD, it is shown that the flame is mainly sensitive to rotational fluctuations, assumed to have their origin in the global swirling motion of the flow. As the burner power is increased further, the pressure spectrum shows a peak around $473 \mathrm{~Hz}$ corresponding to the appearance of a thermoacoustic instability. DMD analysis and computation of a local Rayleigh index show that the flame motion corresponds to an opening of the flame cone in phase with the pressure fluctuation, effectively adding energy to the system and keeping the instability going. Simultaneously, the flame is also still impacted by the previously highlighted rotational fluctuations. Finally, the burner reaches a steady state of high amplitude thermo-acoustic instability at $500 \mathrm{~Hz}$ with an associated flame motion completely similar to the previous one. The rotational motions can no longer be detected in the flame behaviour, probably because of the high amplitude fluctuations induced 
by the thermoacoustic instability. Using Hilbert transform to analyze the temporal evolution of the pressure fluctuations and the global heat release, it is shown that the switch to the $500 \mathrm{~Hz}$ instability happens abruptly which discards a temperature change as the cause for the instability frequency change. Furthermore, the phase difference between pressure and heat release also changes abruptly indicating a modification of the feedback mechanism piloting the instability. Explanations for this might involve non-linear mode switching as well as liquid fuel injection fluctuations.

Despite the use of state of the art diagnostic and post-processing techniques, only limited information can be gathered because of the extremely complex nature of such an experiment. They however give potential explanations for the appearance of thermo-acoustic instabilities in realistic conditions. LES analyzes for a similar operating point provided very useful insight on the instability mechanisms when compared with experimental data [11]. It appears that the complementarity between experiments, numerical simulations and theory is necessary to understand and tackle the combustion instability issues in realistic cases.

\section{ACKNOWLEDGMENT}

The stay of Antoine Renaud at JAXA and Keio University has been supported by the Erasmus Mundus EASED program (Grant 2012-5538/004-001) coordinated by CentraleSupelec.

\section{REFERENCES}

[1] Correa, S. M., 1998. "Power generation and aeropropulsion gas turbines: From combustion science to combustion technology". Symposium (International) on Combustion, 27(2), Jan., pp. 1793-1807.

[2] Candel, S., 2002. "Combustion dynamics and control: progress and challenges". Proceedings of the Combustion Institute, 29(1), pp. 1-28.

[3] Lieuwen, T. C., and Yang, V., 2005. "Combustion instabilities in gas turbine engines(operational experience, fundamental mechanisms and modeling)". Progress in astronautics and aeronautics.

[4] Lefebvre, A. H., 1995. "The Role of Fuel Preparation in Low-Emission Combustion”. Journal of Engineering for Gas Turbines and Power, 117(4), Oct., pp. 617-654.

[5] Kohse-Höinghaus, K., Barlow, R. S., Aldén, M., and Wolfrum, J., 2005. "Combustion at the focus: laser diagnostics and control”. Proceedings of the Combustion Institute, 30(1), Jan., pp. 89-123.

[6] Dhanuka, S. K., Temme, J. E., Driscoll, J. F., and Mongia, H. C., 2009. "Vortex-shedding and mixing layer effects on periodic flashback in a lean premixed prevaporized gas turbine combustor". Proceedings of the Combustion Institute, 32(2), pp. 2901-2908.

[7] Dhanuka, S. K., Temme, J. E., and Driscoll, J. F., 2011. "Lean-limit combustion instabilities of a lean premixed prevaporized gas turbine combustor". Proceedings of the Combustion Institute, 33(2), Jan., pp. 2961-2966.

[8] Stopper, U., Meier, W., Sadanandan, R., Stöhr, M., Aigner, M., and Bulat, G., 2013. "Experimental study of industrial gas turbine 
flames including quantification of pressure influence on flow field, fuel/air premixing and flame shape". Combustion and Flame, 160(10), Oct., pp. 2103-2118.

[9] Temme, J. E., Allison, P. M., and Driscoll, J. F., 2014. "Combustion instability of a lean premixed prevaporized gas turbine combustor studied using phase-averaged PIV". Combustion and Flame, 161(4), Apr., pp. 958-970.

[10] Boxx, I., Slabaugh, C., Kutne, P., Lucht, R. P., and Meier, W., 2015. “3khz PIV/OH-PLIF measurements in a gas turbine combustor at elevated pressure". Proceedings of the Combustion Institute, 35(3), pp. 3793-3802.

[11] Tachibana, S., Saito, K., Yamamoto, T., Makida, M., Kitano, T., and Kurose, R., 2015. "Experimental and numerical investigation of thermo-acoustic instability in a liquid-fuel aero-engine combustor at elevated pressure: Validity of large-eddy simulation of spray combustion”. Combustion and Flame, 162(6), June, pp. 2621-2637.

[12] Yamamoto, T., Shimodaira, K., Kurosawa, Y., and Yoshida, S., 2011. "Combustion Characteristics of Fuel Staged Combustor for Aeroengines at LTO Cycle Conditions". ASME, pp. 951-958.

[13] Yamamoto, T., Shimodaira, K., Yoshida, S., and Kurosawa, Y., 2013. "Emission Reduction of Fuel-Staged Aircraft Engine Combustor Using an Additional Premixed Fuel Nozzle". Journal of Engineering for Gas Turbines and Power, 135(3), Feb., p. 031502.

[14] Sadanandan, R., Stöhr, M., and Meier, W., 2008. "Simultaneous OH-PLIF and PIV measurements in a gas turbine model combustor". pp. 609-618.

[15] Schmid, P. J., 2010. "Dynamic mode decomposition of numerical and experimental data". Journal of Fluid Mechanics, 656, July, pp. 5-28.

[16] Schmid, P. J., 2011. "Application of the dynamic mode decomposition to experimental data". Experiments in Fluids, 50(4), Feb., pp. 1123-1130.

[17] Rowley, C. W., Mezić, I., Bagheri, S., Schlatter, P., and Henningson, D. S., 2009. "Spectral analysis of nonlinear flows". Journal of Fluid Mechanics, 641, Nov., p. 115.

[18] Poinsot, T. J., Trouve, A. C., Veynante, D. P., Candel, S. M., and Esposito, E. J., 1987. "Vortex-driven acoustically coupled combustion instabilities". Journal of Fluid Mechanics, 177(-1), Apr., p. 265.

[19] Palies, P., Durox, D., Schuller, T., and Candel, S., 2010. “The combined dynamics of swirler and turbulent premixed swirling flames". Combustion and Flame, 157(9), Sept., pp. 1698-1717.

[20] Samaniego, J. M., Yip, B., Poinsot, T., and Candel, S., 1993. "Low-frequency combustion instability mechanisms in a side-dump combustor". Combustion and Flame, 94(4), Sept., pp. 363-380.

[21] Richecoeur, F., Hakim, L., Renaud, A., and Zimmer, L., 2012. "DMD algorithms for experimental data processing in combustion". Proceedings of the Summer Program, p. 459.

[22] Moeck, J. P., Bourgouin, J.-F., Durox, D., Schuller, T., and Candel, S., 2012. "Nonlinear interaction between a precessing vortex core and acoustic oscillations in a turbulent swirling flame”. Combustion and Flame, 159(8), Aug., pp. 2650-2668. 
[23] Alekseenko, S. V., Dulin, V. M., Kozorezov, Y., and Markovich, D. M., 2012. "Effect of High-Amplitude Forcing on Turbulent Combustion Intensity and Vortex Core Precession in a Strongly Swirling Lifted Propane/Air Flame". Combustion Science and Technology, 184(10-11), Oct., pp. 1862-1890.

[24] Renaud, A., Ducruix, S., Scouflaire, P., and Zimmer, L., 2015. "Experimental Study of the Interactions Between Air Flow Rate Modulations and PVC in a Swirl-Stabilised Liquid Fuel Burner”. In ASME Turbo Expo 2015: Turbine Technical Conference and Exposition, ASME, pp. V04AT04A063+.

[25] Dowling, A. P., and Morgans, A. S., 2005. "Feedback Control Of Combustion Oscillations". Annual Review of Fluid Mechanics, 37(1), Jan., pp. 151-182.

[26] Ducruix, S., Schuller, T., Durox, D., and Candel, S., 2003. "Combustion Dynamics and Instabilities: Elementary Coupling and Driving Mechanisms". Journal of Propulsion and Power, 19(5), Sept., pp. 722-734.

[27] de la Cruz Garcia, M., Mastorakos, E., and Dowling, A., 2009. "Investigations on the self-excited oscillations in a kerosene spray flame". Combustion and Flame, 156(2), Feb., pp. 374-384.

[28] Noiray, N., Durox, D., Schuller, T., and Candel, S., 2008. “A unified framework for nonlinear combustion instability analysis based on the flame describing function". Journal of Fluid Mechanics, 615, Nov., p. 139.

[29] Matveev, K. I., and Culick, F. E. C., 2003. "A model for combustion instability involving vortex shedding”. Combustion Science and Technology, 175(6), June, pp. 1059-1083.

[30] Apeloig, J. M., d'Herbigny, F.-X., Simon, F., Gajan, P., Orain, M., and Roux, S., 2015. "Liquid-Fuel Behavior in an Aeronautical Injector Submitted to Thermoacoustic Instabilities”. Journal of Propulsion and Power, 31(1), Jan., pp. $309-319$.

[31] Silva, C. F., Nicoud, F., Schuller, T., Durox, D., and Candel, S., 2013. "Combining a Helmholtz solver with the flame describing function to assess combustion instability in a premixed swirled combustor”. Combustion and Flame, 160(9), Sept., pp. 1743-1754. 